\title{
Respostas cardiorrespiratórias do teste de sentar e levantar em indivíduos pré e pós-cirurgia cardíaca: corte transversal
}

\author{
Cardiopulmonary responses of the sit-to-stand test in \\ individuals pre and post-heart surgery: cross section
}

\author{
Eliane Roseli Winkelmann ${ }^{1}$ (i) \\ Luana Gehm da Silva ${ }^{2}$ (1) \\ Mariana Motta Dias da Silva ${ }^{3}$ (1)
}

\author{
Pollyana Windmoller ${ }^{4}$ (1) \\ Audrey Borghi Silva ${ }^{5}$
}

'Autora para correspondência. Universidade Regional do Noroeste do Estado do Rio Grande do Sul (ljuí). Rio Grande do Sul, Brasil. elianew@unijui.edu.br 2Universidade Regional do Noroeste do Estado do Rio Grande do Sul (ljuí). Rio Grande do Sul, Brasil. luana.ks@outlook.com

${ }^{3}$ Universidade Federal de Santa Maria (Santa Maria). Rio Grande do Sul, Brasil. marimotta9@gmail.com ${ }^{4}$ Universidade Regional do Noroeste do Estado do Rio Grande do Sul (ljuí). Rio Grande do Sul, Brasil. pollyana.w@unijui.edu.br 5Universidade Federal de São Paulo (São Paulo). São Paulo, Brasil. audrey@ufscar.br

RESUMO | INTRODUÇÃO: As repercussões cardiorrespiratórias da cirurgia cardíaca podem ser avaliadas por teste submáximo. OBJETIVO: comparar as respostas cardiorrespiratórias do teste de sentar e levantar em um minuto (TSL1) nos indivíduos, entre o momento pré e pós de cirurgia cardíaca. MÉTODOS: Estudo de caráter transversal e analítico, incluiu 45 indivíduos de ambos os sexos, estáveis hemodinamicamente, com fração de ejeção maior que $45 \%$, que foram submetidos à cirurgia de revascularização do miocárdio no Instituto do Coração de um Hospital do interior do estado do Rio Grande do Sul, entre 2018 e 2019. As variáveis de desfechos foram coletadas no repouso e ao final do teste, um dia antes da cirurgia e no pós-operatório: pressão arterial sistólica e diastólica (PAS e PAD em $\mathrm{mmHg}$ ), frequência cardíaca (FC bpm), frequência respiratória ( $F R$ rpm), saturação periférica de oxigênio (SpO2 $\%$ ), fadiga de membros inferiores (Fmm 0-10) e dispneia (Di 0-10), número de repetições e interrupções do teste. Foi utilizado o programa $\mathrm{R}$ para o tratamento dos dados, para avaliar a normalidade foi aplicado o teste de Shapiro Wilk, a comparação dos grupos pelo teste não paramétrico de Wilcoxon. RESULTADOS: A maioria do sexo masculino (71\%) e média de idade foi de $61 \pm$ 9 anos. No pré-operatório, ocorreu aumento entre o repouso e o final do teste, PAS, FC, FR, Fmm e Di $(p<0,05)$. No pós-operatório, houve aumento entre o repouso e o final do teste, para FC, FR, Fmm e Di $(P<0,05)$, contudo, sem elevação da PAS. Ao comparar as variáveis entre os momentos pré e pós, observamos maiores valores da FC, FR e número de interrupções na condição pós $(p<0,05)$, bem como menores valores para a PAS, SpO2 e número de repetições $(p<0,05)$ para essa condição. CONCLUSÃO: Os dados desta pesquisa comprovam que o TSL1 realizado no pré-operatório de cirurgia cardíaca, assim como entre o $4^{\circ}$ ou $5^{\circ}$ dia de pós-cirurgia cardíaca, é seguro e eficaz, representado pela ausência das repercussões cardiorrespiratórias que comprometessem ou agravassem o quadro clínico do paciente. O TSL1 foi capaz de induzir respostas cardiorrespiratórias fisiológicas no pré-operatório; contudo, na condição pós acarretou respostas cardiorrespiratórias mais elevadas no repouso e atenuada resposta em exercício em comparação ao pré-operatório.

PALAVRAS-CHAVE: Fisioterapia. Reabilitação Cardiovascular. Doenças cardiovasculares. Fisiologia. Hemodinâmica. Teste sentar e levantar em um minuto.
ABSTRACT | INTRODUCTION: The cardiorespiratory repercussions of heart surgery can be assessed through submaximal testing. OBJECTIVE: Compare cardiorespiratory responses to the one-minute sit-and-stand test in individuals pre- and post-heart surgery. METHODS: An analytical, cross-sectional study was conducted involving 45 hemodynamically stable male and female patients with an ejection fraction greater than $45 \%$ submitted to coronary artery bypass surgery at a cardiology service of a hospital in the interior of the state of REDACTED between 2018 and 2019. The following variables were collected at rest and the end of the test one day before surgery and postoperatively: systolic and diastolic blood pressure (SBP and DBP, $\mathrm{mmHg}$ ), heart rate ( $\mathrm{HR}$, $\mathrm{bpm})$, respiratory rate (RR, rpm), peripheral saturation oxygen (SpO2, \%), lower limb fatigue (LLF, 0-10), dyspnea (0-10), number of test repetitions and number of interruptions. The R program was used to process the data. The Shapiro-Wilk test was used for the determination of normality. The groups were compared using the non-parametric Wilcoxon test. RESULTS: Most participants were male $(71 \%)$, and the mean age was $61 \pm 9$ years. In the preoperative period, statistically significant increases ( $\mathrm{p} \leq 0.05$ ) were found for SBP, HR, RR, LLF, and dyspnea between resting values and the end of the test. Significant increases $(p \leq 0.05)$ were found in the postoperative period for HR, RR, LLF, and dyspnea between rest and the end of the test, with no increase in SBP. Comparing the preoperative and postoperative evaluations variables, higher $H R$, RR, the number of interruptions and lower SBP, SpO2, and the number of repetitions were found after surgery $(p \leq 0.05)$. CONCLUSION: The one-minute sit-to-stand test induced physiological cardiorespiratory responses in the preoperative evaluation. However, higher cardiorespiratory responses at rest and an attenuated response to exercise were found in the postoperative evaluation compared to the preoperative evaluation.

KEYWORDS: Physiotherapy. Cardiovascular Rehabilitation. Cardiovascular disease. Physiology. Hemodynamics. One-minute sit-to-stand test. 


\section{Introdução}

As doenças cardiovasculares (DCV) são, atualmente, as causas mais comuns de morbidade e a principal causa de mortalidade no mundo. ${ }^{1}$ Avanços terapêuticos, clínicos e cirúrgicos estão em constante evolução para prevenir eventos agudos, alívio dos sintomas, com melhora da qualidade de vida e do prognóstico. A cirurgia cardíaca é altamente complexa e requer cuidados intensivos ${ }^{2}$ e interdisciplinares. Além disso, a reabilitação fisioterapêutica é essencial no período pós-operatório. ${ }^{3}$

Um dos métodos utilizados para a avaliação fisioterapêutica hospitalar para pacientes submetidos à cirurgia cardíaca é o teste de sentar e levantar em um minuto (TSL1). ${ }^{4}$ Este teste avalia a capacidade física funcional e a resistência periférica dos membros inferiores. $\frac{5}{}$ Também permite ser realizado em pouco tempo e, em praticamente qualquer lugar ${ }^{6}$, é considerado seguro. Pelo seu baixo risco cardiovascular, poderá ser adotado na prática clínica. ${ }^{-}$

Os sinais vitais são medidas clássicas da hemodinâmica bem estabelecidas na literatura e importantes ferramentas de diagnóstico. ${ }^{8-11} \mathrm{O}$ estudo de Pinsky et al. traz o monitoramento hemodinâmico funcional como avaliação para as variáveis hemodinâmicas de forma dinâmica em resposta a uma perturbação prédefinida. ${ }^{10}$ Por exemplo, no estudo de García et al..11, os autores observaram que na insuficiência cardiovascular, que é caracterizada por uma má perfusão tecidual quando a frequência cardíaca $(F C)$ aumenta e / ou a pressão arterial (PA) diminuem ao sentar ou levantar, é razoável presumir que existe algum grau de hipovolemia presente. Com isso, é observado que as medidas avaliativas não mudam, porém, há alteração dos valores obtidos em resposta a um desafio fisiológico pré-definido.

O TSL1 é um teste submáximo que permite avaliar, durante um minuto, o número de repetições do ato de sentar e levantar de uma cadeira, sem auxílio dos membros superiores, assim como o número de interrupções durante o teste. É utilizado em várias populações como em indivíduos submetidos a cirurgia cardíaca ${ }^{12}$, doença renal crônica ${ }^{13}$, idosos ${ }^{7}$, tabagistas ${ }^{14}$ e indivíduos hígidos. ${ }^{15}$ Durante este teste também é possível mensurar as respostas hemodinâmicas e respiratórias uma vez que os sinais podem ser coletados no repouso e no final do exercício. ${ }^{7.15} \mathrm{O}$ teste pode ser executado em trinta segundos ${ }^{7,15}$ ou um minuto. ${ }^{4-6,12,13}$ Foi escolhido aplicar o TSL1, pois possui mais evidências cientificas descritas na literatura, e essa avaliação já é rotina na instituição de ensino a qual o estudo pertence, certificando a capacitação da correta aplicabilidade do teste.

O procedimento cirúrgico acarreta alterações das respostas hemodinâmicas e respiratórias, o que pode impactar em alterações nos testes funcionais. Estas respostas já foram descritas na literatura ${ }^{Z, 15}$, mas ainda se percebe a necessidade de estudos em diferentes populações e, atualmente, identificar a faixa etária afetada com as respectivas repercussões, visto o processo de transição epidemiológica e considerando o aumento do envelhecimento, transformações da urbanização e globalização, assim como estilo de vida dos indivíduos..$^{16}$ O presente estudo é relevante, pois analisa as repercussões hemodinâmicas e respiratórias da cirurgia através de um teste de baixo custo para avaliação da capacidade física funcional que pode ser aplicado na prática clínica. Outro aspecto é que não identificamos evidências cientificas que descrevessem esta análise comparando as respostas hemodinâmicas e respiratórias entre os diferentes momentos cirúrgicos, ou seja, entre o pré e pós-operatório de cirurgia cardíaca. Portanto, conhecer estas respostas nos diferentes momentos da avaliação fisioterapêutica no acompanhamento cirúrgico é fundamental para guiar as condutas desses profissionais. Sendo assim, o objetivo era comparar as respostas cardiorrespiratórias do TSL1 nos indivíduos entre o momento pré e pós- cirurgia cardíaca. 


\section{Método}

Trata-se de uma pesquisa com delineamento transversal e analítico, aprovada pelo Comitê de Ética em Pesquisa da Universidade Regional do Noroeste do Estado do Rio Grande do Sul (CAAE: 38912120.1.0000.5350 e parecer $n^{\circ}: 4.400 .333$ ). A coleta de dados ocorreu entre 2018 e 2019. Foram incluídos na amostra 45 indivíduos de ambos os sexos, estáveis hemodinamicamente com fração de ejeção maior que $45 \%$, que foram submetidos ao procedimento cirúrgico cardíaco no Instituto do Coração do Hospital no interior do Estado do Rio Grande do Sul e aqueles que aceitaram participar da pesquisa. Foram excluídos indivíduos que possuíam problemas ortopédicos ou neurológicos, tiveram complicações que prolongassem o período acima de seis dias ou não cumpriram o protocolo de avaliação nos dois momentos.

Para descrever o perfil da amostra do estudo, foram coletadas informações quanto à idade, sexo, presença de diabetes mellitus, histórico de infarto agudo do miocárdio (IAM), hipertensão arterial sistólica (HAS), dislipidemia, doença pulmonar obstrutiva crônica (DPOC) e medidas antropométricas como peso, altura a partir do prontuário hospitalar. Também foram coletadas variáveis intra-hospitalares com relação à cirurgia: clampeamento da aorta (minutos), tempo de circulação extracorpórea (minutos), fração de ejeção do ventrículo esquerdo (percentual), tempo de cirurgia (minutos), tempo de internação na UTI (minutos), tempo de internação no leito (minutos) e tempo de internação hospitalar total (minutos).

O TSL1 foi adaptado pelo descrito no estudo de Ozalevli et al. .5 , no qual, ao sinal de partida, o indiví- duo deve levantar e sentar novamente em uma cadeira, sem auxílio dos braços e repetir o movimento o mais rápido possível e seguro, com o objetivo de realizar o máximo de repetições no período de um minuto. Este teste foi realizado um dia antes da cirurgia e após a cirurgia antes da alta hospitalar $\left(4^{\circ}\right.$ ao $5^{\circ}$ dia do pós-operatório). Também foram coletados os sinais vitais hemodinâmicos e respiratórios no repouso e no final do teste. As variáveis de desfecho foram: pressão arterial sistólica (PAS em $\mathrm{mmHg}$ ) e diastólica (PAD mmHg), frequência cardíaca (FCbpm) e respiratória (FRrpm), saturação periférica de oxigênio (SpO2), sensação de fadiga de membros inferiores (Fmm de 0-10), dispneia (Di de 0-10), número de repetições e número de interrupções.

Para análise estatística dos dados, foi utilizado o programa R (versão 4.0.3). Os dados foram apresentados em valores absolutos e relativos. As variáveis quantitativas foram analisadas através de média e desvio padrão, não sendo aplicado categorização, ou mediana, primeiro e terceiro quartil. Para avaliar a normalidade das variáveis quantitativas e qualitativas, foi aplicado o teste Shapiro Wilk. As comparações dos grupos, para verificar a existência de diferença significativa entre as variáveis, foram realizadas pelo teste não paramétrico Wilcoxon, $p<0,05$.

\section{Resultados}

Foram recrutados 62 indivíduos, sendo excluídos 17 pacientes; a amostra final do estudo foi de 45 indivíduos (Figura 1). 
Figura 1. Fluxograma dos pacientes no pré e pós-operatório de pacientes submetidos a cirurgia cardíaca eletiva

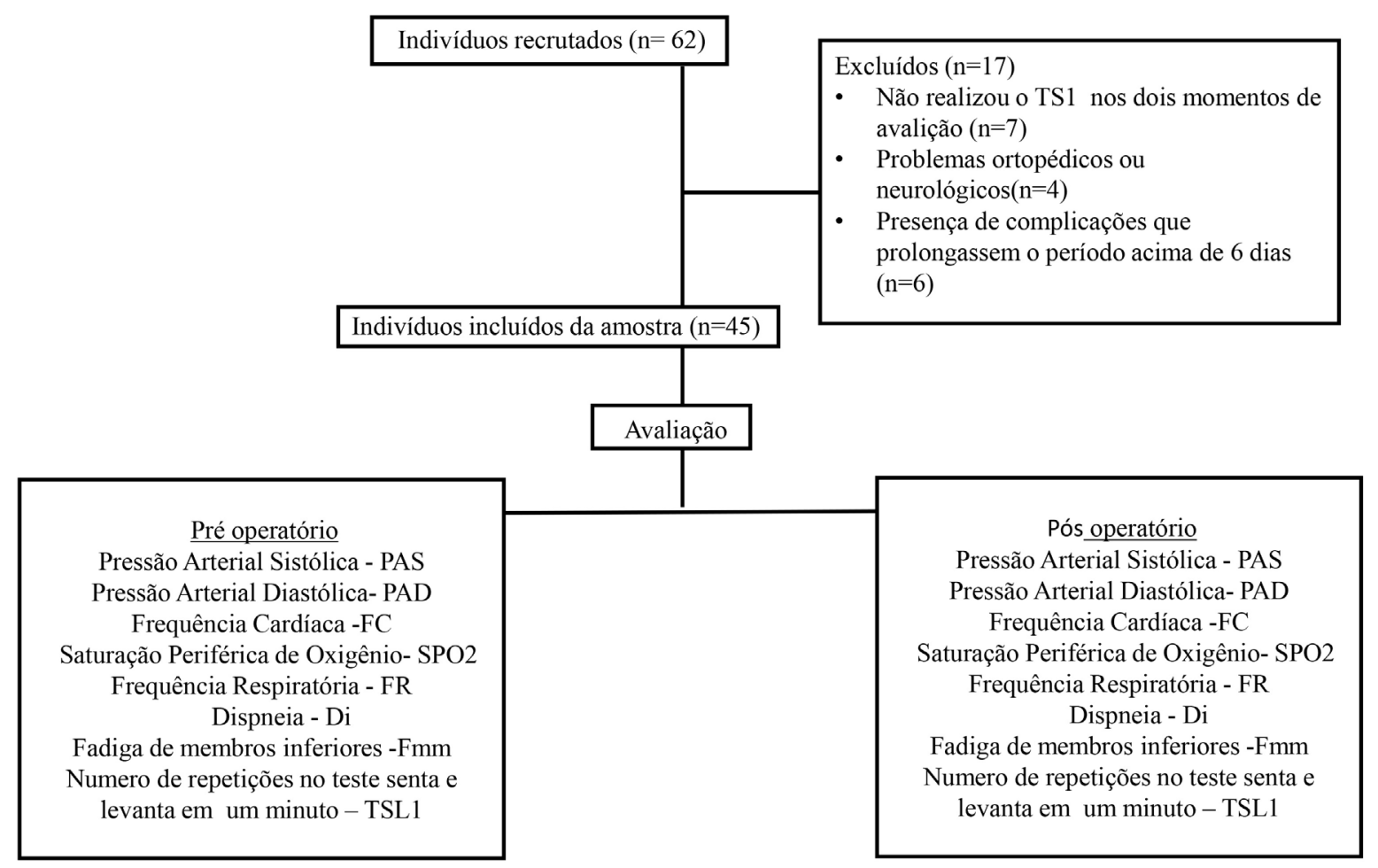

PAS: pressão arterial sistólica; PAD: pressão arterial diastólica; FC: frequência cardíaca; SpO2: saturação periférica de oxigênio; FR: frequência respiratória; Di: dispneia; Fmm: fadiga de membros inferiores

A maioria era do sexo masculino (71\%). A média de idade foi de $61 \pm 9$ anos. Em relação aos fatores de risco, encontrados na amostra a maior frequência de sobrepeso, acometendo $38(84 \%)$ indivíduos. Referente à média de tempo para os procedimentos da cirurgia foi observado uma duração total da cirurgia de $199 \pm 48$ minutos, o clampeamento da aorta foi $61 \pm 15$ minutos e a CEC foi de $73 \pm 17$ minutos. Em relação ao tempo de internação total, a média foi de $7 \pm 1$ dias, (Tabela 1 ).

Tabela 1. Caracterização da amostra e das variáveis clínicas e intra-hospitalar de pacientes submetidos a cirurgia cardíaca eletiva

\begin{tabular}{lccc}
\hline & Masculino & Feminino & Total \\
\hline Número da amostra, $\mathrm{n}(\%)$ & $32(71)$ & $13(29)$ & $45(100)$ \\
Idade, média $\pm \mathrm{DP}$ & $61 \pm 9$ & $62 \pm 9$ & $61 \pm 9$ \\
Fatores de Risco, $\mathrm{n}(\%)$ & & & \\
$\quad$ Diabetes & $17(38)$ & $7(16)$ & $24(53)$ \\
Dislipidemia & $8(18)$ & $2(4)$ & $10(22)$ \\
DPOC & $4(9)$ & $1(2)$ & $5(11)$ \\
IAM & $13(29)$ & $4(9)$ & $17(38)$ \\
ICC & $4(9)$ & $2(4)$ & $6(13)$ \\
Sobrepeso & $28(62)$ & $10(22)$ & $38(84)$ \\
HAS & $21(47)$ & $11(24)$ & $32(71)$ \\
Variáveis intra-hospitalares, média \pm DP & & & \\
Clamp Aorta, min & $61 \pm 13$ & $60 \pm 20$ & $61 \pm 15$ \\
CEC, min & $74 \pm 16$ & $68 \pm 20$ & $73 \pm 17$ \\
Fração de ejeção, \% & $64 \pm 12$ & $67 \pm 10$ & $64 \pm 12$ \\
Tempo UTI, horas & $60 \pm 15$ & $56 \pm 12$ & $59 \pm 14$ \\
Tempo Quarto, horas & $78 \pm 25$ & $76 \pm 14$ & $78 \pm 22$ \\
Tempo Internação, horas & $176 \pm 73$ & $152 \pm 28$ & $169 \pm 64$ \\
Tempo de Cirurgia, min & $207 \pm 51$ & $177 \pm 34$ & $199 \pm 48$ \\
\hline DP: desvio padrão; DPOC: doença pulmonar obstrutiva crônica; IAM: infarto agudo do miocárdio; ICC: insuficiência \\
cardíaca crônica; HAS: hipertensão arterial sistêmica; CEC: circulação extracorpórea; UTI: unidade de terapia intensiva.
\end{tabular}


A tabela 2 apresenta os resultados do TSL1. O pré-operatório demonstrou um aumento estatisticamente significativo do repouso para o final do teste para as variáveis: PAS, FC, FR, Fmm e Dispneia $(p<0,05)$. No pós-operatório, ocorreu um aumento significativo destas variáveis $(p<0,05)$, com exceção da PAS. Não houve alterações na PAD e SpO2 em ambos as fases operatórias. Houve uma redução do número de repetições entre o momento pré (18 repetições) e pós-operatório (10 repetições) estatisticamente significativo $(p \leq 0,001)$. Ao comparar as variáveis entre o pré e pós-operatório, na fase de repouso e no final do teste TSL1, observamos maiores valores da FC, FR $(p<0,05)$ bem como menores valores da PAS, SpO2 e número de repetições no pós-operatório, $p<0,05$.

Tabela 2. Análise das variáveis cardiorrespiratória no TLS1 no pré e pós-operatório de pacientes submetidos a cirurgia cardíaca eletiva

\begin{tabular}{|c|c|c|c|c|c|}
\hline & \multicolumn{2}{|c|}{ Pré $\mathrm{N}=45$} & \multicolumn{2}{|c|}{ Pós $N=45$} & \multirow[b]{2}{*}{$\mathrm{p}$-value } \\
\hline & Mediana e (Q1 - Q3) & $p$-value & Mediana e (Q1 - Q3) & $p$-value & \\
\hline PAS inicial & $130(120-140)$ & \multirow{2}{*}{0.0071 * } & $120(120-130)$ & \multirow{2}{*}{0.127} & $0.0395 *$ \\
\hline PAS final & $140(130-150)$ & & $130(120-140)$ & & $0.0015^{*}$ \\
\hline PAD inicial & $80(80-90)$ & \multirow{2}{*}{0.8040} & $80(70-80)$ & \multirow{2}{*}{0.3264} & 0.0580 \\
\hline PAD final & $80(80-90)$ & & $80(70-90)$ & & 0.2461 \\
\hline FC inicial & $68(63-79)$ & \multirow{2}{*}{$0.0006^{*}$} & $87(79-98)$ & \multirow{2}{*}{$0.0209 *$} & $\leq 0.001$ * \\
\hline FC final & $83(67-94)$ & & $93(87-108)$ & & $0.0008^{*}$ \\
\hline SPO2 inicial & $96(95-97)$ & \multirow{2}{*}{0.6403} & $94(92-96)$ & \multirow{2}{*}{0.4021} & $0.0002^{*}$ \\
\hline SPO2 final & $96(95-98)$ & & $96(92-97)$ & & $0.0069 *$ \\
\hline FR inicial & $17(14-18)$ & \multirow{2}{*}{$\leq 0.001$ * } & $18(15-21)$ & \multirow{2}{*}{$0.0287 *$} & $0.0126 *$ \\
\hline FR final & $19(17-20)$ & & $20(19-23)$ & & $0.0086 *$ \\
\hline Di inicial & $0(0-0)$ & \multirow{2}{*}{$0.0013^{*}$} & $0(0-0)$ & \multirow{2}{*}{$0.0016 *$} & 0.6958 \\
\hline Di final & $0(0-2)$ & & $0(0-2)$ & & 0.7458 \\
\hline Fmm inicial & $0(0-0)$ & \multirow{2}{*}{$0.0175^{*}$} & $0(0-0)$ & \multirow{2}{*}{$0.0366 *$} & 0.9478 \\
\hline Fmm final & $0(0-1)$ & & $0(0-0)$ & & 0.6591 \\
\hline$N^{\circ}$ Repetições & $18(15-22)$ & & $10(7-12)$ & & $\leq 0.001 *$ \\
\hline
\end{tabular}

PAS: pressão arterial sistólica; PAD: pressão arterial diastólica; FC: frequência cardíaca; $\mathrm{SpO}_{2}$ : saturação periférica de oxigênio; FR: frequência respiratória; Di: dispneia; Fmm: fadiga de membros inferiores. Comparações de diferença de média intragrupo (momento inicial e final do teste) e entre grupo (pré e pós) Teste Wilcoxon.

\section{Discussão}

O presente estudo buscou comparar as respostas cardiorrespiratórias no TSL1 nos indivíduos entre o momento pré e pós-cirurgia cardíaca. Os principais resultados neste estudo mostram que a resposta fisiológica esperada para o TSL1, verificado a partir do aumento estatisticamente significativo das variáveis: PAS, FC, FR, Bd e Bp no pré-operatório e aumento dessas variáveis, com exceção da PAS, no pós-operatório. Além disso, evidenciou-se na análise entre o pré e pós-operatório o aumento estatístico para a FC, FR e número de interrupções, uma diminuição dos valores para a PAS, SpO2 e número de repetições.

Em relação ao número de repetições durante o TSL, a mediana obtida em nosso estudo, entre o pré e pós-operatório de cirurgia cardíaca, teve redução significativa, respectivamente de 18 para 10 repetições, corroborando com os estudos de Steffens et al. em que o TSL1 apresentou redução no número de repetições entre estes períodos de 17,98 (pré) para 14,51 (pós) operatório.12 Outros estudos com pacientes submetidos à cirurgia cardíaca apresentam resultados similares, como o estudo de Windmoller et al. que mostra reduções do número de repetições, de 17,38 para 10,83 (grupo reabilitação padrão) e de 18,50 para 13,36 (grupo reabilitação intervenção. ${ }^{4}$ Tanto no pré e pós-operatório, o número de repetições no TSL1 dos pacientes na cirurgia cardíaca é bem inferior ao descrito em outras populações, o que reforça que estes pacientes têm menor desempenho no TSL1. A redução significativa dos valores no pós-operatório, comparada ao pré pode ser atribuída à baixa capacidade de exercício gerado pelo ato cirúrgico, por ser uma cirurgia de grande porte e que leva as alterações significativas. ${ }^{16}$ Outras possíveis causas são a imobilidade no leito e as medicações no pós-operatório. 
Desta forma, este estudo mostra uma redução da resistência muscular periférica, entre pré e pós-operatório de cirurgia cardíaca. Estas alterações, influenciadas pelo procedimento cirúrgico, indicam a necessidade de se mensurar a funcionalidade, antes e após a cirurgia, com a finalidade de conhecer a dinâmica do processo terapêutico e intervir quando necessário, não permitindo que se estabeleça uma limitação funcional. Ozalevli et al. utilizou o TSL1 para avaliar a resistência muscular periférica, em uma população de indivíduos com DPOC, e evidenciou que este teste determina o estado funcional, semelhante ao TC6, porém com a vantagem de apresentar menor estresse hemodinâmico, facilidade e praticidade na aplicação para avaliar ao estado físico do paciente. $\frac{5}{}$

Em relação às respostas cardiorrespiratórias no TSL1 entre o pré e pós-operatório, ocorreu aumento estatístico significativo para a FC, FR e número de interrupções, em contrapartida ocorreu uma diminuição dos valores para a PAS, SpO2 e número de repetições, para o mesmo momento de avaliação. Esses achados podem estar relacionados ao estresse da cirurgia cardíaca, ao uso de medicamentos, imobilidade no leito hospitalar, dificuldade para realizar as trocas gasosas e dor à incisão cirúrgica.

Gottardi et al. avaliaram seis pacientes saudáveis usando o SST de 30 segundos e um minuto e encontraram aumentos nos resultados da FC, PAS e da Escala de Borg com ambas as modalidades, mas nenhum efeito significativo na $\mathrm{SpO} 2$ em indivíduos saudáveis. $\frac{15}{}$ Possivelmente, estes achados estão relacionados com o estresse da cirurgia cardíaca, medicamentos no organismo, imobilidade no leito, dificuldade para executar as trocas gasosas e dor na incisão cirúrgica. Assim como no estudo de Morais et al., o TSL 30 segundos, aplicado em dois grupos de idosos nos quais foi observado no grupo sedentário aumento significativo da PAS (117,3 para 125,3 $\mathrm{mmHg})$ e da FC (75,1 para 94,8 bpm). Contudo, no grupo ativo sofreram aumentos significativos apenas a FC ( 73,7 para 79,6 bpm) e a FR $(16,4$ para 18,8 rpm). ․․ Entretanto, é importante destacar que a resposta da PAS frente aos testes também foi discreta e longe dos valores considerados inadequados pelas diretrizes de saúde (PAS $>220 \mathrm{mmHg}$ ou PAD $>115$ $\mathrm{mmHg}$ ), o que reforça a segurança cardiovascular do teste investigado, sendo esse de baixo risco cardiovascular e podem ser adotados com segurança na prática clínica.
$\mathrm{Na}$ análise das respostas cardiorrespiratórias durante o TSL1 (repouso $x$ final do teste) no pré operatório, ocorreu um aumento estatisticamente significativo do repouso para o final do teste, entre as variáveis: PAS, FC, FR, Bd e Bp. No pós-operatório, ocorreu um aumento destas variáveis, com exceção da PAS, corroborando com os resultados do estudo de Morais et al., no qual foi realizado um estudo de corte transversal, com 30 indivíduos idosos, divididos em grupo ativo e grupo sedentário, evidenciando aumento significativo da PAS $(p<0,001)$ e da FC $(p<0,001)$ no grupo sedentário, contudo, apenas a FR $(p<0,001)$ e a FC $(p=0,003)$ sofreram aumentos significativos no grupo ativo. Aumento da PAS foi observado somente após a realização do teste de sentar e levantar da cadeira, especificamente no grupo sedentário. Tal achado reforça a hipótese de uma adaptação cardiovascular positiva, visto que a resposta pressórica ao esforço também é atenuada frente à prática de exercícios físicos; porém, com o tempo, a tendência é uma regulação autonômica e, consequentemente, a diminuição dessa variável. $\mathrm{O}$ aumento destas variáveis é fisiológico, também foi observado em outros testes como o teste de caminhada de 100 metros. ${ }^{17} \mathrm{O}$ estudo de Cordeiro et al. analisou em 30 indivíduos submetidos a cirurgia cardíaca, variáveis hemodinâmicas durante um protocolo de caminhada de $100 \mathrm{~m}$, em que esta promoveu como resposta imediata um significante aumento da FC $(81,7$ para 94,1 bpm) e da FR (19,4 para 24 rpm) e não evidenciou alterações na PAS, PAD e SpO2. ${ }^{17}$ Visto que o teste de caminhada de 100 metros é considerado leve, os valores apresentados em nosso estudo mantêm o aumento da FC e da FR mesmo em um teste submáximo.

O aumento considerado fisiológico durante o teste e o aumento das variáveis, com exceção da PAS, aliado à diminuição do número de repetições, reflete que o indivíduo consegue expressar neste teste as condições das repercussões da cirurgia cardíaca de grande porte..$_{1,18,19}$ Nesta fase, o indivíduo está mais debilitado, com repercussões da dor no pós-operatória, devido à abertura do tórax, o que repercute na diminuição das condições físicas para realizar o teste. Isto pode também explicar o comportamento da PAS no pós-operatório, pois a mesma aumenta à medida que tem um aumento do esforço. ${ }^{8,18,19}$ Outro aspecto é que o teste não provocou alterações da PAD e SpO2. O esforço físico durante o exercício pode não alterar a PAD.12,20,21 Também, os indivíduos eram cardiopatas, mas sem doença pulmonar prévia observada e descrita nos prontuários o que pode explicar o comportamento de manutenção dos valores durante o teste. $12,19,22,23$ 
Sobre a segurança do TSL1 em pacientes no pré e pós-operatório de cirurgia cardíaca, esta pesquisa comprovou que o TSL1 realizado no pré-operatório de cirurgia cardíaca, assim como entre o $4^{\circ}$ ou $5^{\circ}$ dia de pós-cirurgia cardíaca, é seguro. Não se observou nenhuma alteração que comprometesse a continuidade ou a realização do teste. Nenhum paciente teve que parar o teste por alguma repercussão hemodinâmica e respiratória que levasse algum comprometimento do quadro clínico. O teste mostrou a redução da resistência muscular periférica provocada pela cirurgia. Os resultados sobre a diminuição do número de repetições entre o pré e pós-operatório confirmam este achado.

Os resultados podem ser preditivos de alterações em decorrência da cirurgia cardíaca, sendo também variáveis para prescrição de exercício físico após alta hospitalar e acompanhamento multiprofissional, visto a necessidade de reabilitação fisioterapêutica, com base nas repercussões apresentadas pelo TSL1.

Este estudo trouxe evidências científicas na contribuição do TSL1 para o entendimento da aplicação no pós-operatório de cirurgia cardíaca. Porém, algumas limitações do estudo podem ser descritas como: a pesquisa se realizou em único centro, portanto, os dados não devem ser extrapolados para populações com perfil diferente, além da não identificação do nível de atividade física dos indivíduos e de fumantes na amostra.

\section{Conclusão}

Os dados desta pesquisa comprovam que o TSL1 realizado no pré-operatório de cirurgia cardíaca, assim como entre o $4^{\circ}$ ou $5^{\circ}$ dia de pós-cirurgia cardíaca, é seguro e eficaz, representado pela ausência das repercussões cardiorrespiratórias que comprometessem ou agravassem o quadro clínico do paciente.

\section{Contribuições dos autores}

Silva LG participou da concepção, delineamento, busca e análise estatística dos dados da pesquisa, interpretação dos resultados, redação do artigo científico, revisão e aprovação final do artigo. Silva MMD participou da análise estatística dos dados da pesquisa, interpretação dos resultados. Windmoller $\mathrm{P}$ participou da coleta de dados e revisão final apara aprovação do artigo. Silva $A B$ participou revisão e aprovação final do artigo. Winkelmann ER participou da concepção, delineamento, análise estatística dos dados da pesquisa, interpretação dos resultados, redação, revisão e aprovação final do artigo científico.

\section{Conflitos de interesses}

Nenhum conflito financeiro, legal ou político envolvendo terceiros (governo, empresas e fundações privadas, etc.) foi declarado para nenhum aspecto do trabalho submetido (incluindo, mas não se limitando a subvenções e financiamentos, participação em conselho consultivo, desenho de estudo, preparação de manuscrito, análise estatística, etc.).

\section{Referências}

1.Nascimento BR, Brant LC, Oliveira GM, Malachias MV, Reis GM, Teixeira RA, et al. Epidemiologia das Doenças Cardiovasculares em Países de Língua Portuguesa: Dados do "Global Burden of Disease", 1990 a 2016. Arq. Bras. Cardiol. 2018;110(6):500-11. https://doi.org/10.5935/abc.20180098

2.Soares JM, Costa AE, Pissaia LF. Percepções de enfermeiros sobre Sistematização da Assistência de Enfermagem no pós-operatório de cirurgia cardíaca. Res Soc and Dev. 2019;8(9):e24891278. https://doi.org/10.33448/rsd-v8i9.1278

3. Winkelmann ER, Dallazen F, Bronzatti AB, Lorenzoni JC, Windmöller P. Análise do protocolo adaptado de steps na reabilitação cardíaca na fase hospitalar. Braz J Cardiovasc Surg. 2015;30(1):40-8. https://doi.org/10.5935/1678-9741.20140048

4. Windmöller P, Bodnar ET, Casagrande J, Dallazen F, Schneider J, Berwanger SA, et al. Physical Exercise Combined W/ith CPAP in Subjects /Who Underwent Surgical Myocardial Revascularization: A Randomized Clinical Trial. Respiratory Care [Internet]. 2020;65(2):150-7. Disponível em: http://rc.rcjournal.com/ content/65/2/150.short 
5. Ozalevli S, Ozden A, Itil O, Akkoclu A. Comparison of the Sit-to-Stand Test with 6 min walk test in patients with chronic obstructive pulmonary disease. Respiratory medicine. 2007;101(2):286-93. https://doi.org/10.1016/j.rmed.2006.05.007

6. Gonçalves E, Colet CF, Windmoller P, Winkelmann ER. Correlation of the sit-to-stand test, the walk test and waist circumference before cardiac surgery. Mundo saúde [Internet]. 2019;767-81. Disponível em: http://bvsms.saude.gov.br/bvs/ periodicos/mundo saude artigos/correlation walk cardiac .pdf

7. Morais KA, Pereira MD, Cruz CJ, Drummond A. Respostas cardiovasculares agudas frente a diferentes testes funcionais em idosos. Rev Kairós. 2019;22(1):109-22. https://doi. org/10.23925/2176-901X.2019v22i1p109-122

8.McLean, AS. Editorial: Haemodynamic monitoring: the why, when, which and what. Current opinion in critical care. 2019;25(3), 244-245. https://doi.org/10.1097/MCC.0000000000000606

9. Scheeren TWL, Saugel B. Preface on advances in hemodynamic monitoring in perioperative medicine. Best Pract Res Clin Anaesthesiol. 2019;33(2):125-6. https://doi.org/10.1016/j. bpa.2019.09.001

10.Pinsky MR. Functional hemodynamic monitoring. Crit Care Clin. 2015;31(1):89-111. https://doi.org/10.1016/j.ccc.2014.08.005

11. García X, Pinsky MR. Clinical applicability of functional hemodynamic monitoring. Ann Intensive Care. 2011;1:35. https:// doi.org/10.1186/2110-5820-1-35

12. Steffens É, Dallazen F, Sartori CC, Chiapinotto S, Battisti IDE, Winkelmann ER Condições físico-funcionais e qualidade de vida de pacientes no pré e pós-operatório de cirurgia cardíaca. Rev Pesq Fisio. 2016;6(4). https://doi.org/10.17267/2238-2704rpf. $\underline{\text { v6i4.1149 }}$

13. Fassbinder TR, Winkelmann ER, Schneider J, Wendland J, Oliveira OB. Capacidade funcional e qualidade de vida de pacientes com doença renal crônica pré-dialítica e em hemodiálise-um estudo transversal. J Bras Nefrol. 2015;37(1):4754. https://doi.org/10.5935/0101-2800.20150008

14. Sandi APS, Costa LA, Ferreira MHO, César EP, Carvalho PMM. $O$ desempenho no teste de sentar levantar entre fumantes e não fumantes. ASSOBRAFIR Ciência [Internet]. 2017;8(1):13-22. Disponível em: http://www.uel.br/revistas/uel/index.php/rebrafis/ article/viewFile/26464/21198
15. Gottardi FC, Brizola IM, Maciel PF, Guimarães SF, Alves JF, Antero G, et al. Efeitos do teste Sit-to-stand 30 segundos e 1 minuto sobre os aspectos hemodinâmicos. Perspect. Exp. Clín., Inovações Bioméd. Educ. Saúde [Internet]. 2018;4(2):11. Disponível em: https://periodicos.ufms.br/index.php/pecibes/ article/view/6982

16. Corrêa B, Cardoso DM. Capacidade funcional e estado mental de pacientes submetidos à cirurgia cardíaca. Fisioter. Mov. 2017;30(4):805-11. https://doi.org/10.1590/1980-5918.030.004. A016

17. Cordeiro AL, Amorim NM, Andrade PH, Esquivel MS, Guimarães AR, Melo TA, et al. Alterações fisiológicas da caminhada e tempo de internamento no pós-operatório de cirurgia cardíaca. Int J Cardiovasc Sci [Internet]. 2015;28(5):480-6. Disponível em: http://www.onlineijcs.org/english/sumario/28/pdf/ v28n6a07.pdf

18. Leguisamo CP, Kalil RA, Furlani AP. A efetividade de uma proposta fisioterapêutica pré-operatória para cirurgia de revascularização do miocárdio. Braz. J. Cardiovasc. Surg. 2005;20(2):134-41. https://doi.org/10.1590/S0102$\underline{76382005000200008}$

19. Reis MM, Lima ED, Casagrande RI, Fioresi M, Leite FM, Primo CC. Perfil epidemiológico de pacientes submetidos à cirurgia. Rev. enferm. UFPE on line [Internet]. 2019;13(4):1015-22. Disponível em: https://periodicos.ufpe.br/revistas/revistaenfermagem/ article/download/238020/31797

20. Soares GM, Ferreira DC, Gonçalves MP, Alves TGS, David FL, Henriques KMC, et al. Prevalência das principais complicações pós-operatórias em cirurgias cardíacas. Rev Bras Cardiol [Internet]. 2011;24(3):139-46. Disponível em: http://www. onlineijcs.org/english/sumario/24/pdf/v24n3a02.pdf

21. Strolischein CA, Silva DR, Costa EL, Sancoré FD, Azeredo TG, Fontoura FC. Prevalência das principais complicações pós-operatório em cirurgias cardíacas de revascularização do miocárdio em hospital filantrópico de Cuiabá-MT. Revista da Saúde da AJES [Internet]. 2019;5(9). Disponível em: http://www. revista.ajes.edu.br/index.php/sajes/article/view/305

22.Ricardo DR, Araújo CGS. Reabilitação cardíaca com ênfase no exercício: uma revisão sistemática. Rev Bras Med Esporte. 2006;12(5):279-85. https://doi.org/10.1590/S1517$\underline{86922006000500011}$

23. Strassmann A, Steurer-Stey C, Lana KD, Zoller M, Turk AJ, Suter $P$, et al. Population-based reference values for the 1-min sit-to-stand test. Int J Public Health. 2013;58(6):949-53. https://doi. org/10.1007/s00038-013-0504-z 